\title{
A LINEAR DIOPHANTINE PROBLEM
}

\author{
S. M. JOHNSON
}

1. Introduction. Let $a_{1}, a_{2}, \ldots, a_{t}$ be a set of groupwise relatively prime positive integers. Several authors, $(\mathbf{2} ; \mathbf{3} ; \mathbf{5} ; \mathbf{6})$, have determined bounds for the function $F\left(a_{1}, \ldots, a_{t}\right)$ defined by the property that the equation

$$
n=a_{1} x_{1}+a_{2} x_{2}+\ldots+a_{t} x_{t}
$$

has a solution in positive integers $x_{1}, \ldots, x_{t}$ for $n>F\left(a_{1}, \ldots, a_{t}\right)$. If $F\left(a_{1}, \ldots, a_{t}\right)$ is a function of this type, it is easy to see that

$$
G\left(a_{1}, \ldots, a_{t}\right)=F\left(a_{1}, \ldots, a_{t}\right)-a_{1}-a_{2}-\ldots-a_{t}
$$

is the corresponding function for the solvability of (1) in non-negative $x$ 's.

It is well known that $a_{1} a_{2}$ is the best bound for $F\left(a_{1}, a_{2}\right)$ and $a_{1} a_{2}-a_{1}-a_{2}$ for $G\left(a_{1}, a_{2}\right)$. Otherwise only in very special cases have the best bounds been found, even for $t=3$.

In the present paper a symmetric expression is developed for the best bound for $F\left(a_{1}, a_{2}, a_{3}\right)$ which solves that problem and gives insight on the general problem for larger values of $t$. In addition, some relations are developed which may be of interest in themselves.

2. A General Property. For $t \geqslant 2$, let $B\left(a_{1}, a_{2}, \ldots, a_{t}\right)$ be the best bound for $F\left(a_{1}, a_{2}, \ldots, a_{t}\right)$, that is, $B$ is the maximum number $N$ where

$$
N \neq \sum_{i=1}^{t} x_{i} a_{i} \quad \text { for any } x_{i}>0 .
$$

Then note that $B$ is the maximum $N$ from a restricted set of numbers $N$ satisfying both (3) and

$$
N+a_{i}=\sum_{j=1}^{t} y_{i j} a_{j}, \quad y_{i j}>0 \text { for each } i
$$

since the definition of $B$ implies $B$ satisfies (4). Thus, in particular,

$$
N=\left(y_{11}-1\right) a_{1}+y_{12} a_{2}+\ldots+y_{1 t} a_{t}, y_{1 j}>0 \text {. }
$$

But by (3), $y_{11}-1 \leqslant 0$ so that $y_{11}=1$ since $y_{11}>0$. By symmetry we have

Theorem 1. For every $N$ satisfying (3) and (4) there are representations of $N$ for each $i=1,2, \ldots, t$ of the form

$$
N=\sum_{\substack{j=1 \\ j \neq i}}^{t} y_{i j} a_{j}, \quad y_{i j}>0,
$$

and $B$ is the maximum such $N$.

Received October 21, 1957; in revised form March 9, 1959. 
3. The Case $t=3$. A reduction formula. We seek an expression for $B=B\left(a_{1}, a_{2}, a_{3}\right)$ having the property that (1) is satisfied for $n>B$ but is not satisfied for $n=B$. Let us first reduce the problem to the case of pairwise relatively prime $a$ 's.

Let $d_{i j}=\left(a_{i}, a_{j}\right), a_{i}=b_{i} d_{i j} d_{i k}$, so that $\left(b_{1}, b_{2}\right)=\left(b_{2}, b_{3}\right)=\left(b_{3}, b_{1}\right)=1$. Then we have

Theorem 2.

$$
B\left(a_{1}, a_{2}, a_{3}\right)=d_{12} d_{23} d_{31} B\left(b_{1}, b_{2}, b_{3}\right) .
$$

Proof. First we show that if we write $d=d_{12}, \bar{b}_{1}=d_{13} b_{1}, \bar{b}_{2}=d_{23} b_{2}$ so that $\left(d, a_{3}\right)=\left(\bar{b}_{1}, \bar{b}_{2}\right)=1$, then

$$
B\left(d \bar{b}_{1}, d \bar{b}_{2}, a_{3}\right)=d B\left(\bar{b}_{1}, \bar{b}_{2}, a_{3}\right) .
$$

Suppose that $d B\left(\bar{b}_{1}, \bar{b}_{2}, a_{3}\right)=d \bar{b}_{1} x+d \bar{b}_{2} y+a_{3} z, x, y, z>0$. Then since $(d$, $\left.a_{3}\right)=1$, we must have $z=w d$, $w>0$, so that $B\left(\bar{b}_{1}, \bar{b}_{2}, a_{3}\right)=\bar{b}_{1} x+\bar{b}_{2} y$ $a_{3} w, x, y, w>0$, a contradiction to the definition of $B\left(\bar{b}_{1}, \bar{b}_{2}, a_{3}\right)$. In addition, for any positive integer $m>0$, we show that

$$
d B\left(\bar{b}_{1}, \bar{b}_{2}, a_{3}\right)+m=d \bar{b}_{1} x+d \bar{b}_{2} y+a_{3} z, \quad x, y, z>0 .
$$

We apply a result from (2).

Lemma 1 (Brauer). Let $a$ and $b$ be relatively prime positive integers. Then every positive integer $m$ divisible neither by $a$ nor by $b$ is representable either in the form

$$
m=a u+b v, \quad u>0, v>0,
$$

or

$$
m=a b-a u-b v, \quad b>u>0, a>v>0 .
$$

Letting $d=a$ and $a_{3}=b$ in Lemma 1 , if (9) holds, we have

$$
\begin{aligned}
d B\left(\bar{b}_{1}, \bar{b}_{2}, a_{3}\right)+m & =d\left(B\left(\bar{b}_{1}, \bar{b}_{2}, a_{3}\right)+u\right)+v a_{3} \\
& =d \bar{b}_{1} x+d \bar{b}_{2} y+a_{3}(d z+v)
\end{aligned}
$$

by the definition of $B\left(\bar{b}_{1}, \bar{b}_{2}, a_{3}\right)$, giving (8).

If (10) holds, we have $0<u<a_{3}$, and $0<v<d$, so that

$$
d\left(B\left(\bar{b}_{1}, \bar{b}_{2}, a_{3}\right)+a_{3}-u\right)-v a_{3}=d \bar{b}_{1} x+d \bar{b}_{2} y+(d z-v) a_{3},
$$

for $x, y$, and $(d z-v)>0$, giving (8).

Finally, if $m=u d$, then (8) follows directly. If $m=v a_{3}$, write $m=d a_{3}$ $+(v-d) a_{3}$ giving (8). Thus (7) holds. Applying the method of obtaining (7) twice more gives (6) and Theorem 2.

We have thus reduced the problem to where the $a$ 's are pairwise relatively prime. For the moment let $a_{1}>a_{2}>a_{3}$. If

$$
a_{1}=u a_{2}+v a_{3}, \quad u, v>0,
$$

then $B\left(a_{1}, a_{2}, a_{3}\right)=a_{2} a_{3}+a_{1}$ as Brauer showed in (2). Otherwise

$$
B\left(a_{1}, a_{2}, a_{3}\right)<a_{i} a_{j}+a_{k} \text {. }
$$


4. An expression for $B\left(a_{1}, a_{2}, a_{3}\right)$. We develop a symmetric expression for $B\left(a_{1}, a_{2}, a_{3}\right)$ for the case of pairwise relatively prime $a$ 's where each $a_{i} \neq x a_{\text {, }}$ $+y a_{k}, x>0, y>0$. Later we show that this same form of expression gives the general solution for $t=3$.

Definition. Let $L_{i}=$ the minimum positive $K_{i}$ satisfying

$$
K_{i} a_{i}=v_{i j} a_{j}+v_{i k} a_{k}, \quad v_{i j} \geqslant 0, v_{i k} \geqslant 0, \quad i=1,2,3 .
$$

Such a number exists since $B\left(a_{j}, a_{k}\right)=a_{j} a_{k}<K a_{i}$ for large $K$.

Theorem 3. Given

$$
\left(a_{1}, a_{2}\right)=\left(a_{2}, a_{3}\right)=\left(a_{3}, a_{1}\right)=1
$$

and

$$
L_{i}>1
$$$$
i=1,2,3
$$

and

$$
L_{i} a_{i}=x_{i j} a_{j}+x_{i k} a_{k},
$$

then the $x_{i j}$ are uniquely defined and

$$
x_{i j}>0 .
$$

Since $L_{i}>1$, it follows from (10) and (16) that

$$
a_{i}=a_{j} a_{k}-v_{i j} a_{j}-v_{i k} a_{k}
$$

where $0<v_{i \jmath}<a_{k}, 0<v_{i k}<a_{j}$. Thus $v_{i k} a_{k}+a_{i}=\left(a_{k}-v_{i j}\right) a_{j} \geqslant L_{j} a_{j}$ and so by symmetry

$$
L_{j}<a_{k}, \quad \text { for each } j \neq \mathrm{k} .
$$

If $x_{j i}=0$, then $L_{i} a_{i}=x_{j k} a_{k}$ and by (16) $L_{j}=m a_{k}$, a contradiction to (20). This gives (18). Also the $x_{i j}$ are uniquely determined since if $L_{i} a_{i}=x_{i j} a_{j}$ $+x_{i k} a_{k}=z_{i j} a_{j}+z_{i k} a_{k}$, then by (16) we have $x_{i j}=z_{i j}+m a_{k}$ and $x_{i k}=z_{i k}-m a_{j}$. If $m>0, x_{i j}>a_{k}$. But then for some $d>0, L_{i} a_{i}=\left(a_{k}+d\right) a_{j}+x_{i k} a_{k}$ and by (19) we get $\left(L_{i}-1\right) a_{i}=\left(d+v_{i j}\right) a_{j}+\left(x_{i k}+v_{i k}\right) a_{k}$, contradicting the definition of $L_{i}$. Similarly, for $m<0$.

For $t=3$ and (16) and (17) we show that there are just two numbers $N$ with properties (3) and (4) so that $B$ is the larger of these numbers. From (5) such a number $N$ has representations of the form

$$
N=y_{i j} a_{\jmath}+y_{i k} a_{k}
$$

$$
i=1,2,3 \text {. }
$$

Next observe that from (18) we have

$$
y_{i j} \leqslant L_{j}
$$

since otherwise for some $d_{j}>0$ we would have $N=\left(L_{j}+d_{j}\right) a_{j}+y_{i k} a_{k}$ $=x_{j i} a_{i}+d_{j} a_{j}+\left(x_{j k}+y_{i k}\right) a_{k}$, contradicting (3). From (20) and (21) we have

$$
y_{k j}<a_{k}, \quad y_{k j}<a_{i}
$$


Next we show that the representations $\left(5^{\prime}\right)$ for $N$ are unique for each $i$. For otherwise $y_{k i} a_{i}+y_{k j} a_{j}=z_{k i} a_{i}+z_{k j} a_{j}$ and from (16) and (22), $y_{k j}-z_{k j}$ $=m a_{i}, m \leqslant 0$, and $y_{k i}-z_{k i}=m a, m \geqslant 0$, so that $m=0$ and $y_{k j}=z_{k j}$, etc. From $\left(5^{\prime}\right)$ and Theorem 1 we now have unique representations of $N$ of the form

$$
N=y_{k i} a_{i}+y_{k j} a_{j}=y_{i j} a_{j}+y_{i k} a_{k}=y_{j k} a_{k}+y_{j i} a_{i} .
$$

If $y_{k j}=y_{i j}$, then $y_{k i}=m a_{k}$, contradicting (22). Thus either $y_{k j}<y_{i j}$ or $y_{k j}>y_{i j}$.

Case 1. If

$$
y_{k j}<y_{i j}
$$

then $y_{k i} a_{i}=\left(y_{i \jmath}-y_{k j}\right) a_{j}+y_{i k} a_{k}$ so that $y_{k i} \geqslant L_{i}$. Thus by (21) we have

$$
y_{k i}=L_{i} \text {. }
$$

Then by (24) and $\left(5^{\prime}\right)$

$$
N=L_{i} a_{i}+y_{k j} a_{j}=y_{j i} a_{i}+y_{j k} a_{k}
$$

or $\left(L_{i}-y_{j i}\right) a_{i}+y_{k j} a_{j}=y_{j k} a_{k}$, where $L_{i} \geqslant y_{j i}$ by (21). If $L_{i}=y_{j i}$ then $y_{k j}=m a_{k}$, contradicting (22), so that $L_{i}-y_{j i}>0$ and $y_{j k} \geqslant L_{k}$ by the definition of $L_{k}$. But then $y_{j k}=L_{k}$ by (21). Thus (23) implies that $y_{k i}=L_{i}$, $y_{j k}=L_{k}$, and cyclically, $y_{i j}={ }^{2} L_{j}$. But then by $\left(15^{\prime}\right)$

$$
N=\left(x_{i j}+y_{k j}\right) a_{j}+x_{i k} a_{k}=L_{j} a_{j}+y_{i k} a_{k}
$$

and by the uniqueness of these representations and by cyclic permutation of subscripts, we have

$$
y_{i k}=x_{i k}
$$

and

$$
L_{\jmath}=x_{i j}+x_{k \jmath} .
$$

Thus if $y_{k j}<y_{i j}$, we get a unique number $N$ where

$$
N=L_{i} a_{i}+x_{k j} a_{j}
$$

with cyclic permutations of subscripts.

\section{Case 2. If}

$$
y_{k j}>y_{i j}
$$

we get another number where by symmetry

$$
N^{\prime}=L_{i} a_{i}+x_{j k} a_{k}
$$

with cyclic permutations of subscripts. $N \neq N^{\prime}$ since otherwise $x_{j k} a_{k}=x_{k j} a_{j}$ which implies $x_{j k} \geqslant a_{j}$, which by (25) contradicts (22). Note that these two 
numbers are the only numbers with properties (3) and (4) for (16), (17), and $t=3$. Since $B$ is the largest number with property (3), it satisfies (t) so that $B$ is the maximum of $N$ and $N^{\prime}$ and we have

THEorem 4. Given (16) and (17), then for cyclic permutations of subscripts

$$
B\left(a_{1}, a_{2}, a_{3}\right)=L_{i} a_{i}+\max \left(x_{k j} a_{j}, x_{j k} a_{k}\right)
$$

and (26) holds.

Also it is easy to verify that $C$, the corresponding best bound for $G\left(a_{1}, a_{2}, a_{3}\right)$, satisfies

$$
C\left(a_{1}, a_{2}, a_{3}\right)+a_{1}+a_{2}+a_{3}=B\left(a_{1}, a_{2}, a_{3}\right) .
$$

5. A computing algorithm for $L_{i}$ and $x_{i j}$. Thus we have shown that finding $B$ is equivalent to finding the set of positive integers $L_{i}$ and $x_{i j}$ exhibited in the form of a matrix of detached coefficients of the three equations $\left(15^{\prime}\right)$ as follows:

\begin{tabular}{lll}
\hline$a_{1}$ & $a_{2}$ & $a_{3}$ \\
\hline \hline$-L_{1}$ & $x_{12}$ & $x_{13}$ \\
$x_{21}$ & $-L_{2}$ & $x_{23}$ \\
$x_{31}$ & $x_{32}$ & $-L_{3}$ \\
\hline
\end{tabular}

In order to develop a simple computing algorithm for these numbers, we need the following result.

Lemma 2. Given $\left(a_{1}, a_{2}\right)=\left(a_{2}, a_{3}\right)=\left(a_{3}, a_{1}\right)=1$, then any system of integers $K_{i}>1$ and $v_{i j}>0$ (not necessarily $L_{i}$ and $x_{i j}$ ) satisfying (15) and (26) $K_{i}=v_{\jmath i}+v_{k i}$, implies that

$$
K_{i} K_{j}-v_{i j} v_{j i}=v_{j i} v_{k j}+v_{k i} K_{j}=\lambda a_{k} \geqslant a_{k}
$$

for some positive integer $\lambda$.

If we write

$$
v_{j k}\left(K_{i} a_{i}-v_{i j} a_{j}\right)=v_{i k} v_{j k} a_{k}=v_{i k}\left(K_{j} a_{j}-v_{j i} a_{i}\right),
$$

then

$$
\left(v_{j k} K_{i}+v_{i k} v_{j i}\right) a_{i}=\left(v_{i k} K_{j}+v_{j k} v_{i j}\right) a_{j}
$$

and (32) follows by (16) and (26).

Furthermore, we have

TheoreM ${ }^{7}$. If (16) and (17) hold, then the $L_{i}$ and $x_{i j}$ in Theorem 4 are characterized by the equations $\left(15^{\prime}\right)$ and (26), and

$$
L_{i} L_{j}+x_{i j} x_{j i}=a_{k},
$$

for cyclic permutations of subscripts. That is, $\lambda=1$ in (32). 
Proof. Suppose a system of $K_{i}$ and $v_{i j}$ satisfy (15), (26), and (33) where at least one $K_{i}>L_{i}$, the minimum positive integer satisfying (15).

Case 1. If $K_{1}=L_{1}, K_{2}=L_{2}$, then $K_{3}=L_{3}$ by $(26)$ and Theorem 3 .

Case 2. Suppose $K_{1}=L_{1}$, but $K_{2}>L_{2}, K_{3}>L_{3}$.

Then $x_{12}=v_{12}$ and $x_{13}=v_{13}$ by Theorem 3 and by (15), (26), and (33) $a_{1}=K_{2} K_{3}-v_{32} v_{23}=K_{2} K_{3}-\left(K_{2}-x_{12}\right)\left(K_{3}-x_{13}\right)=x_{12} K_{3}+x_{13} K_{2}-$ $x_{12} x_{13}>x_{12} L_{3}+x_{13} L_{2}-x_{12} x_{13}=L_{2} L_{3}-x_{32} x_{23} \geqslant a_{1}$ by (32), a contradiction to the assumption that $K_{2}>L_{2}, K_{3}>L_{3}$.

Case 3. If $K_{1}>L_{1}, L_{2}>K_{2}, K_{3}>L_{3}$, then first observe that either $v_{i j}>x_{i j}$ or $v_{i k}>x_{i k}$, but not both. For suppose $v_{i j}>x_{i j}$ and $v_{i k}>x_{i k}$. By (33) $v_{i j} v_{j k}+K_{j} v_{i k}=a_{i} \leqslant x_{i j} x_{j k}+L_{j} x_{i k}$ by (32). Thus $v_{j k}<x_{j k}$. Similarly $v_{i j} K_{k}+v_{i k} v_{k j}=a_{i} \leqslant x_{i j} L_{k}+x_{i k} x_{k j}$ so that $v_{k j}<x_{k j}$. But then $a_{i} \leqslant L_{j} L_{k}$ $-x_{j k} x_{k j}<K_{j} K_{k}-v_{j k} v_{k j}=a_{i}$, a contradiction.

In addition either $v_{j i}>x_{j i}$ or $v_{k i}>x_{k i}$ but not both. For suppose $v_{1 i}>x_{j i}$ and $v_{k i}>x_{j i}$. By the previous remark $v_{j k}<x_{j k}, v_{k j}<x_{k j}$, leading to the same contradiction obtained above. Thus either $v_{12}, v_{23}, v_{31}$, or $v_{21}, v_{32}, v_{13}$ are larger than the corresponding $x$ 's. That is $v_{i j}>x_{i j}$ for cyclic permutations of subscripts.

Suppose $v_{21}, v_{32}, v_{13}$ are larger than $x_{21}, x_{32}, x_{13}$ respectively. Then by $(26)$

$$
\left(K_{2}-L_{2}\right) a_{2}+\left(x_{23}-v_{23}\right) a_{3}=\left(v_{21}-x_{21}\right) a_{1} \geqslant L_{1} a_{1}
$$

by the definition of $L_{1}$. Thus $v_{21}>L_{1}$ and by cyclic permutation of subscripts $v_{32}>L_{2}, v_{13}>L_{3}$.

Finally $a_{3} \leqslant L_{1} L_{2}-x_{12} x_{21}<L_{1} L_{2}<v_{21} v_{32}<v_{21} v_{32}+K_{2} v_{31}=a_{3}$, a contradiction.

Thus $\lambda=1$ in (32) implies that $K_{i}=L_{i}, v_{i j}=x_{i j}$.

Conversely, $\lambda=1$ in (32), for $K_{i}=L_{i}, v_{i j}=x_{i j}$ etc. By the following computing algorithm we can always find sets of $K_{i}$ and $v_{i j}$ with $\lambda=1$ in (32). Thus they are the desired $L_{i}$ and $x_{i j}$. Moreover since the $x_{i j}$ are unique by Theorem $3, \lambda$ is unique and must equal 1 .

The usefulness of Theorem 5 is apparent since it will be easier to find $K$ 's and $v$ 's satisfying (15), (26), and (33) rather than find minimal solutions to (15).

The algorithm follows. First we solve for any $a_{k}$ in terms of $a_{i}$ and $a_{j}$; for instance, for $k=3$, giving

$$
v_{21} a_{1}-K_{2} a_{2}+a_{3}=0
$$

with $0<v_{21}<a_{2}, 0<K_{2}<a_{1}$ by (10), easily done for example as in (4).

Next construct

$$
-K_{1} a_{1}+v_{12} a_{2}+v_{13} a_{3}=0
$$

where

$$
v_{13}=\left[\frac{a_{1}}{K_{2}}\right], \quad K_{1}=a_{2}-v_{21} v_{13}, a_{1}=K_{2} v_{13}+v_{12}
$$


so that $\lambda=1$ in (32). If $K_{1}>v_{21}$, then $K_{1}=L_{1}, K_{2}=L_{2}$, and $L_{3}$ can be found by (26). Then apply Theorem 4 for $B\left(a_{1}, a_{2}, a_{3}\right)$. If $K_{1} \leqslant v_{21}$, note that $K_{1} \nmid v_{21}$. For if $K_{1} \mid v_{21}$, then since $K_{1}=a_{2}-v_{21} v_{13}, K_{1} \mid a_{2}$. But then in (34) $K_{1} \mid a_{3}$. Thus $\left(a_{2}, a_{3}\right) \geqslant K_{1}>1$, by (17) a contradiction.

Therefore if $K_{1}<v_{21}$ we can construct another equation

$$
\left(v_{21}-p K_{1}\right) a_{1}-\left(K_{2}-p v_{12}\right) a_{2}+\left(1+p v_{13}\right) a_{3}=0
$$

with

$$
p=\left[\frac{v_{21}}{K_{1}}\right] .
$$

Since $v_{21}-p K_{1}>0, K_{2}^{\prime}=K_{2}-p v_{12}$ forms a smaller value of $K_{2}$ in (34).

Note that the pair of equations giving the smallest values of $K_{1}$ and $K_{2}$ will still give $\lambda=1$ in (32). At each stage we repeat the above generating of a smaller $K_{1}$ or $K_{2}$ until eventually $K_{1}=L_{1}, K_{2}=L_{2}$. By Theorem 5 this will come about when we obtain equations of the type (34) and (35) with $K_{1}>v_{21}$ and $K_{2}>v_{12}$.

To illustrate we find $B(137,251,256)$. First calculate that

$$
a_{1}-75 a_{2}+73 a_{3}=0 \text {. }
$$

Then by the algorithm we obtain

$$
\begin{aligned}
3 a_{1}+31 a_{2}-32 a_{3}=0 \\
7 a_{1}-13 a_{2}+9 a_{3}=0 \\
17 a_{1}+5 a_{2}-14 a_{3}=0
\end{aligned}
$$

Thus the matrix of detached coefficients is

\begin{tabular}{rrr}
\hline$a_{1}$ & $a_{2}$ & $a_{3}$ \\
\hline \hline-24 & 8 & 5 \\
7 & -13 & 9 \\
17 & 5 & -14 \\
\hline
\end{tabular}

and $B=24 a_{1}+9 a_{3}=5,592$.

It should be pointed out that solving for (34) is not always necessary. Many computational short cuts become apparent after some practice. Note that the suggested algorithm is not merely numerical but gives algebraic relations as well, enabling one to solve all previously solved special cases for $t=3$ by a unified approach. For example, see the end of the next section.

6. Extensions and restatement of basic theorem. Even if $L_{3}=1$, the statement of Theorems 4 and 5 still holds, dropping the minimality condition on the $L_{i}$. In this case, $B=a_{1} a_{2}+a_{3}$, see (2). But the matrix of coefficients is 


\begin{tabular}{crr}
\hline$a_{1}$ & $a_{2}$ & $a_{3}$ \\
\hline$-a_{2}$ & $a_{1}$ & 0 \\
$a_{2}-x_{31}$ & $-a_{1}-x_{32}$ & 1 \\
$x_{31}$ & $x_{32}$ & -1 \\
\hline
\end{tabular}

with $x_{31}<a_{2}$. Then $\lambda=1$, so that Theorem 5 gives the same result $a_{1} a_{2}+a_{3}$.

Next we show that Theorems 4 and 5 hold even though the $a_{i}$ 's are not reduced to a pairwise relatively prime set $b_{1}, b_{2}, b_{3}$.

We compare the $L$ 's and $x_{i j}$ 's associated with $a_{1}, a_{2}, a_{3}$ with those $L$ 's and $x_{i j}$ 's associated with $b_{1}, b_{2}, b_{3}$. From $\left(15^{\prime}\right), L_{i} a_{i}=x_{i j} a_{j}+x_{i k} a_{k}$, we see that $d_{j k}\left|L_{i}, d_{i j}\right| x_{i k}, d_{i k} \mid x_{i j}$. Thus, setting $L_{i}=d_{j k} L_{i}{ }^{\prime}, x_{i k}=d_{i j} x_{i k}{ }^{\prime}$, we have

$$
L_{j} L_{k}-x_{j k} x_{k j}=a_{i} \text { if and only if } L_{j}^{\prime} L_{k}^{\prime}-x_{j k}^{\prime} x_{k j}^{\prime}=b_{i},
$$

since $d_{i j} d_{i k}\left(L_{j}^{\prime} L_{k}{ }^{\prime}-x_{j k}{ }^{\prime} x_{k j}{ }^{\prime}\right)=d_{i j} d_{i k} b_{i}=a_{i}$.

Finally, all these results can be collected in the following form:

Theorem 6. For $\left(a_{1}, a_{2}, a_{3}\right)=1$, define $B$ to be the largest number not of the form $x a_{1}+y a_{2}+z a_{3}, x, y, z>0$. Then for cyclic permutation of subscripts

$$
B=L_{i} a_{i}+\max \left(x_{j k} a_{k}, x_{k j} a_{j}\right) \text {, }
$$

where

$$
L_{i} a_{i}=x_{i j} a_{j}+x_{i k} a_{k}, \quad L_{i}>0, x_{i j} \geqslant 0, x_{i k} \geqslant 0, \quad L_{i}=x_{j i}+x_{k i}
$$

and

$$
L_{i} L_{j}-x_{i j} x_{j i}=a_{k} .
$$

The $L$ 's and $x$ 's can be found either by the computing algorithm discussed in $\S 5$, modified to solve first for $d_{i j} a_{k}$ in terms of $a_{i}$ and $a_{j}$, or by first applying Theorem 2.

In conclusion, observe that the special cases previously obtained for $t=3$ can be derived directly from the results of this paper.

Example. We can extend the results stated in (5) for $B(a, a+1, a+z)$. Write $a=k z-u, 0 \leqslant u<z, k \geqslant 1, z \geqslant 2$. Then for $u \leqslant k+1$ the coefficient matrix is

\begin{tabular}{ccc}
\hline$a=a_{1}=k z-u$ & $a_{2}=k z-u+1$ & $a_{3}=k z-u+z$ \\
\hline \hline$-(z+k-u)$ & $z-u$ & $k-1$ \\
$z-1$ & $-z$ & 1 \\
$k+1-u$ & $u$ & $-k$ \\
\hline
\end{tabular}

If $u \leqslant 1$, then

$$
B=L_{3} a_{3}+x_{12} a_{2}=\left(\frac{a+u}{z}\right)(a+z)+(z-u)(a+1) .
$$


To correspond to the notation of (5), we solve for $C+1=B+1-\sum a_{i}$. Then

$$
C+1=\left(\frac{a+u}{z}\right) a+(z-2-u) a .
$$

If $u>1$, then $B=L_{3} a_{3}+x_{21} a_{1}=k(a+z)+(z-1) a$, and

$$
C+1=\left[\frac{a+1}{z}\right](a+z)+(z-3) a
$$

since

$$
\left(\frac{a+u}{z}\right)=\left[\frac{a+1}{z}\right]+1
$$

For $t>3$, Theorem 1 holds and the author has verified that relations analogous to Theorem 4 hold in many cases. However, this will be the subject of a later paper.

\section{REFERENCES}

1. P. T. Bateman, Remark on a recent note on linear forms, Amer. Math. Monthly, 65 (1958). 517-518.

2. A. T. Brauer, On a problem of partitions-I, Amer. J. Math., 64 (1942), 299-312.

3. A. T. Brauer and B. M. Seelbinder, On a problem of partitions-II, Amer. J. Math., 66 (1954), 343-346.

4. R. J. Levit, A minimum solution for a diophantine equation, Amer. Math. Monthly, 63 (1956), 646-651.

5. J. B. Roberts, Note on linear forms, Proc. Amer. Math. Soc. (1956), 465-469.

6. - On a diophantine problem, Can. J. Math., 9 (1957), 219-223.

The Rand Corporation

Santa Monica, California 